\title{
Parteienproporz, Länderproporz und Bundestagsgröße im neuen Bundestagswahlrecht
}

\author{
Valentin Schröder
}

Lob für das neue Bundeswahlgesetz beschränkt sich bislang vorwiegend darauf, dass es durch den Ausgleich von Überhangmandaten den Proporz zwischen den Bundestagsparteien sichert und damit zumindest den Vorgaben des Bundesverfassungsgerichts genüge. ${ }^{1}$ Umso ausgiebiger ist die Kritik²; besonders gerügt wird das Gesetz für seine massive Tendenz, den Bundestag zu vergrößern. Unter Umständen kann sich die Sitzzahl um bis zu 200 über die gesetzliche Mandatszahl von 598 hinaus erhöhen. ${ }^{3}$

Nun hat dies aber auch einen Vorteil, denn die zusätzlichen Mandate dienen als Puffer für den Fall parteiinterner Proporzverzerrungen. Das neue Wahlgesetz gleicht bei der Mandatszuteilung an die Parteien - der Oberverteilung - nämlich zwar Abweichungen vom Proporz zwischen den Parteien aus; aber innerhalb der Parteien, also bei der Zuteilung der Mandate an die einzelnen Landeslisten in der Unterverteilung, werden Überhangmandate einer Partei in einem Land nun mit Listenmandaten dieser Partei in anderen Ländern verrechnet. ${ }^{4}$ Dieser Wechsel vom Proporzverfahren in der Oberverteilung zu einem Vorrangverfahren (Direktmandate vor Listenmandate) in der Unterverteilung ist ebenfalls kritisch angemerkt worden ${ }^{5}$, weil die Kompensation von überzähligen Direktmandaten einer Partei in einem Land mit Listenmandaten dieser Partei in anderen Ländern bei Parteien mit Überhangmandaten den innerparteilichen Proporz gleich doppelt verzerrt: erstens durch Mandatszuschüsse für Landesverbände, die nur durch Direktmandate im Bundestag vertreten sind und zweitens durch Mandatsabzüge für Landesverbände, die vorwiegend über Lis-

1 So zum Beispiel Niels Dehmel / Eckhard Jesse, Das neue Wahlgesetz zur Bundestagswahl 2013: Eine Reform der Reform ist unvermeidlich, in: ZParl, 44. Jg. (2013), H. 1, S. 201 - 213, S. 205; Florian Grotz, Happy End oder endloses Drama? Die Reform des Bundestagswahlsystems, in: Eckhard Jesse / Roland Sturm (Hrsg.), Bilanz der Bundestagswahl 2013: Voraussetzungen, Ergebnisse, Folgen, Baden-Baden 2014, S. 113 - 140, S. 137; Gerd Strohmeier, Kein perfektes Wahlsystem, aber ein guter Kompromiss - unter schwierigen Rahmenbedingungen, in: Zeitschrift für Politik, 60. Jg. (2013), H. 2, S. 144 - 161, S. 154.

2 Vgl. zusammenfassend Joachim Behnke, Das neue Wahlgesetz im Test der Bundestagswahl 2013, in: ZParl, 45. Jg. (2014), H. 1, S. 17 - 37.

3 Vgl. ebenda, S. 35 f.; Niels Dehmel / Eckhard Jesse, a.a.O. (Fn. 1), S. 207; Florian Grotz, a.a.O. (Fn. 1), S. 128; Hans Meyer, Das Bundestagswahlrecht 2013, in: Der Bürger im Staat, 63. Jg. (2013), H. 3, S. 208 - 217, S. 214; Friedrich Pukelsheim / Matthias Rossi, Imperfektes Wahlrecht, in: Zeitschrift für Gesetzgebung, 28. Jg. (2013), H. 3, S. 209 - 226, S. 215; Gerd Strohmeier, a.a.O. (Fn. 1), S. 160; Philipp Weinmann, Führt das Wahlrecht zur „Aufblähung“ des Bundestages? Simulationsrechnungen auf Basis des neuen Bundeswahlgesetzes, in: ZParl, 44. Jg. (2013), H. 4, S. $719-741$, S. 739.

4 Für eine Darstellung am Beispiel vgl. Bundeswahlleiter, Wahl zum 18. Deutschen Bundestag am 22. September 2013, Informationen des Bundeswahlleiters, H. 3: Endgültige Ergebnisse nach Wahlkreisen, Wiesbaden 2013, S. 335 f.

5 Vgl. zum Beispiel Hans Meyer, a.a.O. (Fn. 3), S. 213; zur Problematik solcher Kompensationsmodelle vgl. bereits Joachim Behnke, Ein sparsames länderproporzminimierendes parteienproporzgewährendes automatisches Mandatszuteilungsverfahren mit Ausgleich ohne negatives Stimmengewicht, in: ZParl, 43. Jg. (2012), H. 3, S. 675 - 693, S. 685. 
tenmandate im Bundestag vertreten sind. ${ }^{6}$ Besonders „kleine“ Landesverbände sind von der sich öffnenden Schere betroffen, denn bei ihnen genügen ja schon ein oder zwei Mandate für ein Anschwellen oder eben Versiegen ihrer Bundestagsvertretung. ${ }^{7}$ Da selbst bei CDU und SPD auf die Mehrheit aller Landesverbände in der Regel deutlich weniger als zehn Mandate entfallen, kann sich diese überhangmandatsbedingte parteiinterne Umverteilung deshalb stärker auf die Bundestagsrepräsentanz der einzelnen Landesverbände auswirken als das (Zweitstimmen-)Wahlergebnis selbst. Diese Neuerung im Bundestagswahlrecht könnte daher eine beachtliche Sprengkraft für das interne Gefüge derjenigen Bundestagsparteien entfalten, die zahlreiche Direktmandate erzielen und in mehr als einem Bundesland antreten - also mindestens für CDU und SPD, und damit für die beiden nach wie vor weitaus mandatsstärksten Parteien. Die eingangs genannte und oft kritisierte Tendenz zur Bundestagsvergrößerung wirkt dem entgegen.

Weder die Größenordnung der Verzerrung durch das Vorrangverfahren bei der Unterverteilung noch der Grad, zu dem diese Verzerrung mit zusätzlichen Mandaten abgemildert wird, sind bislang näher untersucht worden. Sie sind aber die wichtigsten Konsequenzen der Wahlrechtsnovelle für die Mandatsverteilung innerhalb der Parteien. Hier soll daher zum einen ermittelt werden, wie groß der Umfang der parlamentarischen Proporzverzerrung innerhalb der beiden großen Parteien unter dem neuen Wahlrecht ist. Zum anderen geht es darum, diese Verzerrung als Herausforderung für die Einhaltung der normativen Grundlagen des deutschen Wahlsystems zu untersuchen und Möglichkeiten aufzuzeigen, wie sie zumindest weitgehend bewältigt werden kann, ohne dadurch das Problem der Bundestagsvergrößerung noch zu verschärfen.

\section{Repräsentationsprinzipien und Bundestagsgröße im deutschen Mischwahlsystem}

Florian Grotz identifiziert drei Repräsentationsprinzipien im deutschen Wahlsystem: „personalisiert-territoriale Repräsentation (in Form von Wahlkreismandaten), innerparteilich-föderalen Proporz (in Form von Landeslisten) und nationalen Parteienproporz (durch Errechnung der Gesamtmandate auf Bundesebene) “8. Diese drei Prinzipien sind schon für sich genommen als Zielsetzungen nicht generell zu erreichen. So können etwa Verzerrungen im innerparteilich-föderalen Proporz - also ein Anteil der Bundestagsmandate eines Landesverbands an allen Mandaten seiner Partei, der vom Anteil der Zweitstimmen der entsprechenden Landesliste an allen Stimmen dieser Partei abweicht - im kleinen Umfang schon durch Rundung bei der Mandatszuteilung vorkommen. Je nach Ausgestaltung des Wahlrechts können solche Einschränkungen bei der Umsetzung eines Prinzips aber vor allem durch die

6 Vgl. Daniel Lübbert / Felix Arndt / Friedrich Pukelsheim, Proporzwahrende Anpassung der Bundestagsgröße: Ein Lösungsvorschlag für das Problem der negativen Stimmgewichte bei Bundestagswahlen, in: ZParl, 42. Jg. (2011), H. 2, S. 426 - 435, S. 427.

7 Nur rein numerisch sind die Landeslisten in großen Bundesländern besonders betroffen, vgl. Joachim Behnke, a.a.O. (Fn. 2), S. 34.

8 Vgl. Florian Grotz, Wahlsystem und Regierbarkeit: Das deutsche Wahlsystem auf dem Prüfstand, in: Gerd Strohmeier (Hrsg.), Wahlsystemreform, Sonderband der ZPol, Baden-Baden 2009, S. 155 - 182, S. 173; vgl. auch Franz Urban Pappi / Michael Herrmann, Überhangmandate ohne negatives Stimmengewicht: Machbarkeit, Wirkungen, Beurteilung, in: ZParl, 41. Jg. (2010), H. 2, S. 260 - 278, S. 269 f.; Gerd Strohmeier, a.a.O. (Fn. 1), S. 155 f. 
Umsetzungsweise der anderen Prinzipien massiv auftreten. Würde das Wahlgesetz zum Beispiel nur die Herstellung des nationalen Parteienproporzes (im Folgenden kurz: „Parteienproporz") in der Oberverteilung festschreiben und folglich auf Überhangmandate einer Partei allein durch Ausgleichsmandate für die anderen Parteien reagieren, dann wären bei Parteien mit einem ausgeprägten Überhang sehr starke Verzerrungen des innerparteilichföderalen Proporzes (im Folgenden: „Länderproporz“) unvermeidlich. Bei der Wahl 2009 wären beispielsweise in der CDU/CSU-Bundestagsfraktion die drei Landesverbände BadenWürttemberg, Saarland und Sachsen um je mehr als 20 Prozent über- und die beiden von Brandenburg und Hamburg um rund 20 Prozent unterrepräsentiert. Weitere acht wären um je rund zwölf Prozent über- oder unterrepräsentiert. Nur drei CDU-Landesverbände Bremen, Hessen und Mecklenburg-Vorpommern - wären noch entsprechend ihrem Anteil an allen CDU-Zweitstimmen vertreten; letztere beide nur rein zufällig, weil die dortigen Direktmandatssummen dem innerparteilichen Proporz entsprachen. ${ }^{9}$

Die Ursache solcher Verzerrungen beim Länderproporz ist das unsystematische Zusammenspiel von Verhältnis- und Mehrheitswahlkomponente im Bundestagswahlrecht als Mischwahlsystem. ${ }^{10}$ So sollen einerseits durch die Verhältniswahl mit der Zweitstimme Parteienproporz und Länderproporz im Bundestag hergestellt werden. Andererseits wird durch die Umsetzung des personalisiert-territorialen Repräsentationsprinzips per Mehrheitswahl mit der Erststimme schon rund die Hälfte aller Mandate vergeben. Die Mehrheitswahl selbst wirkt aber zwischen den Parteien erst einmal proporzverzerrend, den anderen Prinzipien also gerade gegenläufig, und dies umso mehr, je geringer der pro Mandat erforderliche Stimmenanteil im Wahlkreis ist. ${ }^{11}$ Zur Herstellung des Proporzes werden deshalb im geltenden Wahlrecht die von jeder Partei in der Mehrheitswahlkomponente erzielten Direktmandate mit den ihr jeweils laut Verhältniswahlkomponente zustehenden Mandaten verrechnet.

9 Diese Berechnung wendet das Verfahren der Direktmandatsorientierten Proporzanpassung an (vgl. Richard Pfeifer / Daniel Lübbert / Kai-Friederike Oelbermann / Friedrich Pukelsheim, Direktmandatsorientierte Proprozanpassung: Eine mit der Personenwahl verbundene Verhältniswahl ohne negative Stimmengewichte, in: Deutsches Verwaltungsblatt, 127. Jg. (2012), H. 12, S. 725 - 730), jedoch ohne den dort vorgeschlagenen zehnprozentigen Mandatsaufschlag für bestimmte Parteien. In der Beispielrechnung hier ergeben sich insgesamt 647 Mandate, zu denen es durch den Ausgleich von drei CSU-Überhangmandaten kommt.

$10 \mathrm{Zu}$ dieser Klassifikation vgl. Joachim Behnke, Das Wahlystem der Bundesrepublik Deutschland: Logik, Technik und Praxis der Verhältniswahl, Baden-Baden 2007, S. 182; André Blais / Louis Massicotte, Electoral Systems, in: Pippa Norris / Lawrence LeDuc / Richard G. Niemi (Hrsg.), Comparing Democracies: Elections and Voting in Global Perspective, Thousand Oaks 1996, S. 49 - 81, S. 54; David M. Farrell, Electoral Systems, Basingstoke 2001, S. 98 f.; Alan Renwick, The Politics of Electoral Reform, Cambridge 2010, S. 150.

$11 \mathrm{Zu}$ der so genannten „Kubusregel“ als Ursache für diese Proporzverzerrungen im Aggregat vgl. Philip Manow, The Cube Rule in a Mixed Electoral System: Disproportionality in German Bundestag Elections, in: West European Politics, 34. Jg. (2011), H. 4, S. 773 - 794; zu ihrer entsprechend massiven empirischen Relevanz für grundlegenden Wahlsystemreformen vgl. Ernesto Calvo, The Competitive Road to Proportional Representation: Partisan Biases and Electoral Regime Change under Increasing Party Competition, in: World Politics, 61. Jg. (2009), H. 2, S. 254 - 295; und für eine kritische Diskussion ihrer Effekte auf die Einhaltung des Parteienproporzes vgl. Eckhard Jesse, Wahlrecht zwischen Kontinuität und Reform: Eine Analyse der Wahlsystemdiskussion und der Wahlrechtsänderungen in der Bundesrepublik Deutschland 19491983, Düsseldorf 1985, S. 184 ff. 
Dadurch wird aber nur der Parteienproporz sichergestellt, denn bei den von der Verrechnung besonders betroffenen Parteien (CDU, CSU und SPD) fallen im Durchschnitt zwei Drittel der Mandate als Direktmandate an. Durch Verrechnung steht ihnen ein entsprechend kleinerer Mandatsanteil für die Landeslisten zur Verfügung. Nun dienen die Listenmandate aber nicht nur der Herstellung des Parteienproporzes, sondern sind auch das einzige Instrument zur Herstellung des Länderproporzes. Je weniger Mandate auf die einzelnen Landeslisten einer Partei verteilt werden können, desto weniger stehen zur Verfügung, um diejenigen Landesverbände zu bedienen, die weniger Direktmandate erzielt haben, als es ihrem Anteil am Zweitstimmenergebnis dieser Partei entspricht. Dieser Engpass kann wiederum zu Einschränkungen bei der Einhaltung des Länderproporzes führen, wenn eine Partei in einem Bundesland mehr Direktmandate gewinnt, als ihr dort laut ihrem Zweitstimmenanteil zustehen, das heißt, wenn außerdem Überhangmandate anfallen. Dann erhält eine Partei ja im jeweiligen Bundesland nicht nur mehr Direktmandate, als sie dort im Vergleich zu den anderen Parteien beanspruchen kann, sondern auch auf den Landesverband dieser Partei entfallen mehr Mandate, als ihm gemessen an seinem Anteil an den Zweitstimmen im Vergleich zu den anderen Landesverbänden seiner eigenen Partei zustehen. Die Verrechnung der Direktmandate reduziert im gleichen Maß, wie Überhangmandate anfallen, außerdem das Mandatskontingent, das an diese anderen Landesverbände verteilt werden kann. Da die Sitzzahl einer Partei insgesamt zudem von ihrem Zweitstimmenanteil abhängt, Überhangmandate aber gerade dann massiv auftreten, wenn dieser besonders niedrig ist und damit besonders wenig Mandate auf ihre Landeslisten verteilt werden können, kann die Herstellung des Länderproporzes bei einer Partei dann je weniger gelingen, desto mehr Überhangmandate bei ihr anfallen. Es gibt für die betroffenen Parteien dann schlicht zu wenig Verteilungsmasse aus der Verhältniswahlkomponente für den Ausgleich von Mandatsüberschüssen einzelner Landesverbände aus der Mehrheitswahlkomponente. Daraus resultiert die schon genannte doppelte Verzerrung. Sie kommt durch die Bevorzugung von Parteienproporz und Personenwahl gegenüber dem Länderproporz zustande.

Der einzige Weg, diese Verzerrung generell aufzulösen, wäre die Vermehrung der Mandate für die davon betroffenen Parteien und damit, entlang des Parteienproporzes, auch der insgesamt zu vergebenden Sitze. Genau dieser Weg ist jedoch nicht bis in die Größenordnung hinein gangbar, die für die generelle Auflösung dieser Verzerrung nötig wäre. ${ }^{12}$ Folglich ergibt sich ein Zielkonflikt zwischen der Einhaltung der drei Repräsentationsprinzipien auf der einen Seite und einer möglichst sparsamen Bundestagsgröße - gemessen an der Einhaltung der gesetzlichen Sitzzahl - auf der anderen.

Dieser Zielkonflikt aus Einhaltung der gesetzlichen Mandatszahl und umfassender Umsetzung der drei Prinzipien wohnt dem deutschen Wahlsystem schon von jeher inne. ${ }^{13} \mathrm{Be}-$

12 Vgl. neben den eingangs Genannten insbesondere Martin Fehndrich, Stellungnahme anlässlich der öffentlichen Anhörung des Innenausschusses am 14. Januar 2013 in Berlin, in: Innenausschuss des Deutschen Bundestags, Drs. 17(4)634 C vom 14. Januar 2013, S. 2.

13 Vgl. Friedrich Pukelsheim, Bundeswahlgesetzt - nächste Etappe, in: Deutsches Verwaltungsblatt, 123. Jg. (2008), S. 889 - 897, S. 889, dagegen interpretieren Pappi und Herrmann sowie Strohmeier einen Teil der resultierenden Divergenzen gerade als Chance für die Gewährung eines systemimmanenten Mehrheitsbonus, während Behnke und Grotz darin wiederum gerade eine Gefahr für die Umsetzung des Parteinproporzes sehen; vgl. Franz Urban Pappi / Michael Herrmann, a.a.O. (Fn. 8), S. 271 f.; Gerd Strohmeier, Die Geister, die Karlsruhe rief: Eine Replik 
reits bei der ersten Bundestagswahl 1949 kam es beispielsweise in Gestalt eines SPD-Überhangmandats in Bremen zu solch zweifacher Proporzverzerrung: Nicht nur war die SPD dadurch gegenüber den anderen Parteien (marginal) überrepräsentiert, sondern auch der Bremer SPD-Landesverband wurde innerhalb der SPD-Bundestagsfraktion so (leicht) bevorteilt. Für die längste Zeit war dieser Zielkonflikt aber kaum beobachtbar, denn es fielen trotz der zunehmenden elektoralen Konzentration des deutschen Parteiensystems seit den 1950er Jahren zwar oftmals Überhangmandate an; aber es waren so wenige, dass weder der Parteienproporz noch der Länderproporz dadurch spürbar verzerrt wurden. Nicht zuletzt deshalb ließ sich das empirische Erscheinungsbild des deutschen Wahlsystems bis in die 1990 er Jahre hinein auch als „not [...] a mixed system, but proportional representation“ 14 beschreiben.

Mit dem säkularen Niedergang der Stimmenanteile für SPD und CDU/CSU und in der Folge dem Anfallen von Überhangmandaten in nennenswerten Größenordnungen - seit 1994 fast immer im zweistelligen Bereich und oftmals zugunsten nur einer Partei - ist die Wirkungsweise des deutschen Wahlsystems als Mischwahlsystem seit geraumer Zeit aber empirisch klar sichtbar. Gerade weil es „vom rechts- und politikwissenschaftlichen Mainstream [...und vom] Bundesverfassungsgericht in allen einschlägigen Urteilen durchgehend als Verhältniswahlsystem charakterisiert" 15 wird, zeigt sich hier der Unterschied zwischen dem Anspruch an das Ergebnis einer Mandatsverteilungsprozedur (dem Proporz zwischen und innerhalb der Parteien) und ihrer Wirkungsweise - die dieses Ergebnis im Rahmen der gesetzlichen Sitzzahl eben nicht sicherstellt. In $\$ 6$ des geltenden Wahlgesetzes wird dieser Unterschied gerade durch die kryptischen Formulierungen genau jener Passagen deutlich, mit denen Parteienproporz und Länderproporz in Übereinstimmung gebracht werden sollen, ohne dass die Konsequenzen dieses Unterfangens für die Bundestagsgröße im Wortlaut deutlich werden.

\section{Parteienproporz und Länderproporz}

Ein prima facie naheliegender Ausweg aus dem beschriebenen Zielkonflikt wäre es, den Länderproporz als eines dieser Prinzipien einfach aufzugeben, um wenigstens die beiden anderen im Rahmen der angestrebten sparsamen Bundestagsgröße umsetzen zu können. Genau dort setzt etwa Hans Meyer an, indem er dem Proporz zwischen den Parteien „emi-

auf die Beiträge zur Wahlsystemreform in Heft 2/2010 der ZParl, in: ZParl, 42. Jg. (2011), H. 1, S. 186 - 193, S. 187; Joachim Behnke / Florian Grotz, Das Wahlsystem zwischen normativer Begründung, empirischer Evidenz und politischen Interessen. Ein Kommentar zu Gerd Strohmeier sowie Franz Urban Pappi und Michael Herrmann, in: ZParl, 42. Jg. (2011), H. 2, S. 419 - 425, S. $422 \mathrm{f}$.

14 Eckhard Jesse, Elections: The Federal Republic of Germany in Comparision, New York u.a. 1992, S. 71.

15 Joachim Behnke / Florian Grotz, a.a.O. (Fn. 13), S. 420. Diese Charakterisierung hat das Bundesverfassungsgericht in seinem 2012er Wahlrechtsurteil bezeichnenderweise nicht daran gehindert, eine Parteienproporzverzerrung durch bis zu 15 Überhangmandate zu akzeptieren, vgl. Bundesverfassungsgericht, Urteil des Zweiten Senats vom 25. Juli 2012 - 2 BvF 3//1, 2 BvR 2670/11, $2 \mathrm{BvE}$ 9/11, in: Mitglieder des Bundesverfassungsgerichts, Entscheidungen des Bundesverfassungsgerichts, Bd. 131, Tübingen 2012. Es liegt auf der Hand, dass 15 Mandate bei entsprechend knappem Wahlausgang ein äußerst wirksamer „Mehrheitsbonus“ sein können. 
nente[...] staatspolitische Bedeutung “ zuspricht, dem Proporz der Landeslisten untereinander aber eben „nur parteipolitische Bedeutung “16. Nun sind die Bundestagsabgeordneten zwar Mitglieder des unitarischen Vertretungsorgans des deutschen Volks und nicht einer Versammlung von „Landesvölkern“; aber sie werden innerhalb der Landesverbände der Parteien nominiert. Dieser dezentrale Nominierungsweg spiegelt wiederum die föderale Struktur der Bundesrepublik wider - und dies gerade bei CDU und SPD. ${ }^{17}$ Und zumindest bei der CDU mit ihrem ideologisch nach wie vor stark entlang der Landesverbände variierenden Profil ${ }^{18}$ dürfte eine Verzerrung des Länderproporzes umso größere Verwerfungen für das Handeln ihrer gesamten Fraktion im Bundestag nach sich ziehen, je ausgeprägter diese Verzerrung ist. Denn aufgrund der Abhängigkeit des Direktmandatskontingents einer Partei auch vom Wahlerfolg zumindest ihrer Hauptkonkurrentin in der Mehrheitswahlkomponente besteht nur ein sehr loser Zusammenhang zwischen (Zweitstimmen-)Wahlerfolg und Länderproporz. So ging 2009 ein recht geringfügiger Rückgang des CDU-Zweitstimmenanteils mit massiven Verlusten der SPD und in der Folge einem drastischen Anstieg der CDU-Direktmandate einher. Je stärker die Direktmandate von Wahl zu Wahl zwischen den Parteien fluktuieren, desto erratischer würde sich bei Beachtung nur des Parteienproporzes das inhaltliche Profil der Parlamentarier derselben Partei von Wahl zu Wahl ändern - je nachdem wie die parteiinterne Verrechnung der Direktmandate sich auf die einzelnen Landesverbände auswirkt. Die Ursache dafür wäre aber gerade nicht das Wählervotum, etwa als Reaktion auf eine programmatische Neuorientierung der gesamten Partei, sondern dieses Profil würde von der Verteilung der Direktmandate über das Bundesgebiet abhängen und damit buchstäblich entlang der Trennlinie zwischen Mehrheits- und Verhältniswahlkomponente verlaufen. Diese Trennlinie wird durch das Wahlgesetz gezogen und nicht in einem Prozess innerparteilicher Willensbildung. Auf letztere kommt es aber an, da die bundesdeutsche Verfassungswirklichkeit maßgeblich vom politischen Profil der Bundestagsparteien geprägt wird.

Wenn außerdem der „Lohn“ eines Landesverbands in Bundestagssitzen nennenswert von der Mandatsart - Direkt- oder Listenmandat - abhängig wäre, würde es für seine Parlamentarier zudem eben nicht nur darauf ankommen, ihre „Mühen“ für das politische Profil der Partei aufzuwenden, sondern auch für eine explizite eigene Profilierung im Wahlkreis. Eine solche Hinwendung zum politischen Unternehmertum der „pork barrel politics“ würde die repräsentative Demokratie in Deutschland wiederum deutlich verändern, ironischerweise genau in eine Richtung, die besonders für Mehrheitswahlsysteme typisch ist. ${ }^{19}$ Darum hat die zumindest ungefähre Einhaltung des Länderproporzes durchaus auch „staatspolitische“ Bedeutung - und deshalb lohnt es sich auch zu untersuchen, inwieweit er eingehalten wird.

Zuvor ist aber noch zu fragen, ob die Einhaltung des Länderproporzes für die Parlamentarier selbst überhaupt relevant ist. So beklagt etwa Grotz „in der Wahlrechtsnovelle logi-

16 Beide Zitate aus Hans Meyer, a.a.O. (Fn. 3), S. 213.

17 Vgl. Udo Zolleis / Josef Schmid, Die Christlich Demokratische Union Deutschlands (CDU), in: Oskar Niedermayer (Hrsg.), Handbuch Parteienforschung, Wiesbaden 2013, S. 415 - 437, S. 427; Tim Spier / Ulrich von Alemann, Die Sozialdemokratische Partei Deutschlands (SPD), in: ebenda, S. 439 - 467, S. 447.

18 Vgl. zum Beispiel Arijana Neumann, Die CDU auf Landesebene: Politische Strategien im Vergleich, Wiesbaden 2012, S. 243.

19 Vgl. etwa John M. Carey, Legislative Voting and Accountability, Cambridge 2009. 
sche Kohärenzprobleme, die offenbar darauf zurückzuführen sind, dass die politisch Verantwortlichen die Neuregelung selbst nicht bis ins letzte Detail durchdrungen haben. So gewährleistet das neue Wahlsystem zwar die Proportionalität zwischen den parlamentarischen Parteien, nicht aber jene zwischen den Landeslisten ein- und derselben Partei - obwohl dies in der Gesetzesbegründung behauptet wird "20. Eine solche Nachlässigkeit könnte darauf hindeuten, dass die Abgeordneten als Vertreter der Parteien selbst keinen besonderen Wert auf die Einhaltung des Länderproporzes legen. Dann wäre dieses Prinzip selbst wiederum nur ein historisches Artefakt und mithin gegebenenfalls verzichtbar.

Dass das neue Wahlrecht den Länderproporz nicht gewährleistet, bedeutet aber noch nicht, dass es ihn auch unmöglich macht oder gravierend einschränkt. Für die Parteien kommt es ja nur darauf an, ob und in welcher Größenordnung das Wahlrecht eine Verzerrung auch tatsächlich bewirkt. Eine Verzerrung des Länderproporzes durch Kompensation von Überhangmandaten mit Listenmandatsansprüchen konnte bei der Beratung des Wahlgesetzes innerhalb der Gesetzgebungskoalition nur CDU und SPD betreffen, denn nur sie könnten nach Lage der Dinge parteiintern wirksame Überhangmandate erzielen. Zumindest die Unionsfraktion erkannte während der Beratungen aber durchaus die „Akzeptanzprobleme [...], die etwa bei einer alternativen Lösung über die Verrechnung von Überhangmandaten hätten entstehen können, weil dann einzelne Landesverbände in Gefahr gewesen wären, stark unterrepräsentiert zu sein " 21 . Und gerade die $\mathrm{CDU}^{22}$ setzte im neuen Wahlgesetz den Dreischritt der Verteilungsprozedur durch, bei der zuerst Mandatskontingente in den 16 Ländern gebildet, diese dann für jede Partei aufsummiert werden und schließlich entlang der in der Summe am meisten überrepräsentierten Partei erst der Parteienproporz hergestellt wird. Dieser Dreischritt ist im geltenden Wahlrecht die Ursache der Erhöhung des CDU-Mandatskontingents, mit der wiederum parteiinterne Verzerrungen ausgeglichen werden können, die aber eben auch zu der beklagten Bundestagsvergrößerung führt. Der Kommentar der CDU-Vertreter im Ausschussbericht deutet durchaus auf ein Verständnis dieses Zusammenhangs zwischen Parteienproporz, Länderproporz und Mandatszahl hin, denn dort heißt es weiter: „Die nun gefundene Ausgleichslösung liege in der Konsequenz des Urteils des Bundesverfassungsgerichts. Die damit einhergehende Möglichkeit einer Vergrößerung des Bundestages sei zwar nicht wünschenswert, aber unvermeidlich." Es besteht also kein Anlass anzunehmen, dass die derzeitigen Regelungen gewissermaßen durch ein Versehen zustande kamen, geschweige denn, dass der Länderproporz den Parlamentariern gleichgültig wäre. So verschroben die Formulierungen im Wahlgesetz auch sein mögen, entspricht ihre Stoßrichtung doch dem Anliegen einer zumindest ungefähren Einhaltung des Länderproporzes. Und dies ist, wie oben argumentiert, in der repräsentativen Demokratie auch legitim.

Unklar konnte bei Verabschiedung des Gesetzes noch sein, wie sich die neuen Regelungen auf die Einhaltung des Länderproporzes genau auswirken würden. Aber dass die „Mög-

20 Florian Grotz, a.a.O. (Fn. 1), S. 129.

21 Deutscher Bundestag, Beschlussempfehlung und Bericht des Innenausschusses (4. Ausschuss) zu dem Gesetzentwurf der Fraktionen CDU/CSU, SPD, FDP und Bündnis 90/Die Grünen „Entwurf eines 22. Gesetzes zur Änderung des Bundeswahlgesetzes“ und zu dem Gesetzentwurf der Abgeordneten Halina Wawzyniak, Dr. Dagmar Enkelmann, Jan Korte, weiterer Abgeordneter und der Fraktion Die Linke. „Entwurf eines ... Gesetzes zur Änderung des Bundeswahlgesetzes“, Drs. 12417 vom 20. Februar 2013, S. 8.

22 So Hans Meyer, a.a.O. (Fn. 3), S. 201. 
lichkeit" einer Bundestagsvergrößerung auch zur Wirklichkeit werden würde - und dass damit auch eine gewisse Verfügungsmasse von Mandaten für die Annäherung an den Länderproporz vorhanden sein würde - war schon im Oktober 2012 einer Modellrechnung des Bundeswahlleiters zu entnehmen. ${ }^{23}$ Dort wird demonstriert, dass es unter dem neuen Wahlrecht bei den Wahlen seit 1994 immer zur Bundestagsvergrößerung gekommen wäre. Die Bundestagsvergrößerung 2013 fügt sich in diese Modellrechnung zumindest aus CDUSicht ein. ${ }^{24}$ Aus den 631 Mandaten für die Oberverteilung ergab sich für sie ein Anspruch auf 255 unterzuverteilende Sitze bei 198 eigenen Direktmandaten, und damit 57 Listenmandate. Diese recht hohe Zahl begrenzte die Abweichung vom Länderproporz auf insgesamt drei Sitze weniger für Baden-Württemberg und Nordrhein-Westfalen und je einen zu viel für Brandenburg, Sachsen-Anhalt und Thüringen. ${ }^{25}$

Diese Abweichungen mögen klein erscheinen. Sie ereigneten sich 2013 aber vor dem Hintergrund eines außerordentlich guten CDU-Wahlergebnisses und damit nur drei Überhangmandaten, die auch den Länderproporz verzerren konnten. ${ }^{26}$ Bei einem weniger eindrucksvollen Wahlausgang (und folglich weniger zu verteilenden Listenmandaten) wären sie wesentlich massiver, wie in den Bubble-Plots in Abbildung 1 gezeigt wird.

Für diese Abbildung wurden die Unterverteilungen für die Landesverbände von CDU und SPD bei den Bundestagswahlen 1949 bis 2013 simuliert. Die beiden oberen Plots geben die Ergebnisse bei einer Verteilung an, die nur den Parteienproporz und den Erhalt aller Direktmandate sicherstellt. Dort können also mehr Mandate verteilt werden als gesetzlich vorgesehen, aber nur so viel mehr, wie für den Parteienproporz erforderlich sind. Die beiden unteren Plots sind Simulationen genau entsprechend dem 2013 geltenden Wahlgesetz. ${ }^{27}$ Für alle Simulationen wurde als Ausgangswert für die Bundestagsgröße die jeweilige

23 Vgl. Bundeswahlleiter, Modellrechnungen für die Bundestagswahlen 1994-2009 (Sitzkontingente mit Ausgleich) vom 9. Oktober 2012; Gz. W/39910010-B56001, Wiesbaden 2012.

24 Die Modellrechnungen des Bundeswahlleiters gingen seinerzeit noch davon aus, dass die Landeskontingente für die Erste Stufe der Verteilung der doppelten Wahlkreisanzahl vor Ort entsprechen würden und nicht dem Anteil jedes Bundeslandes an der deutschen Bevölkerung. Dieser Unterschied führt für die Zeit seit der Wahlkreisreform zu einer einmaligen Überschätzung der Bundestagsvergrößerung in den Modellrechnungen um zwei (für die Wahl 2002).

25 Wäre es zu keiner Bundestagsvergrößerung gekommen, wären unter den dann 242 CDU-Parlamentariern vier Landesverbände mit je einem Mandat unterrepräsentiert gewesen (zusätzlich noch Niedersachsen und Hessen) und vier überrepräsentiert (zusätzlich noch das Saarland).

26 Insgesamt erhielt die CDU vier Überhangmandate, von denen eines (aus dem Saarland) durch die Mandatserhöhung für alle Parteien im Zuge der Herstellung des Parteienproporzes als Verzerrungsquelle für den Länderproporz entfiel.

27 Für die Simulation des nur den Parteienproporz wahrenden Regimes (obere zwei Grafiken) wurde das Pukelsheim-3-Verfahren angewendet, allerdings ohne den dortigen Zehn-Prozent-Aufschlag auf die Direktmandate für bestimmte Parteien. Um die Vergleichbarkeit der Angaben entlang der sonstigen Wahlregeln zu gewährleisten, wurden dabei die heute geltenden Mandatshürden (fünf Prozent der Zweitstimmen bundesweit oder mindestens drei Direktmandate) angewendet und stets erst eine Oberverteilung und danach die Unterverteilungen für CDU und SPD durchgeführt. Dadurch ergeben sich kleine Abweichungen vom seinerzeitigen Ergebnis für die zur Oberverteilung zuteilungsberechtigten Parteien für die Wahlen 1949 und 1953 (in den Simulationen nicht zuteilungsberechtigt für 1949: DtKP/DReP, SSW, WAV; für 1953: Zentrum; für 1990: Bündnis 90, PDS). Berücksichtigt sind durchgehend Direktmandate für Einzelbewerber und für Parteien, die nicht zuteilungsberechtigt waren (1949: drei Mandate für Einzelbewerber, 1953: ein Mandat für das Zentrum, 1990: ein Mandat für die PDS, 2002: zwei Mandate für die PDS). Diese Mandate wurden von den Kontingenten zur Verteilung nach Parteienproporz in 


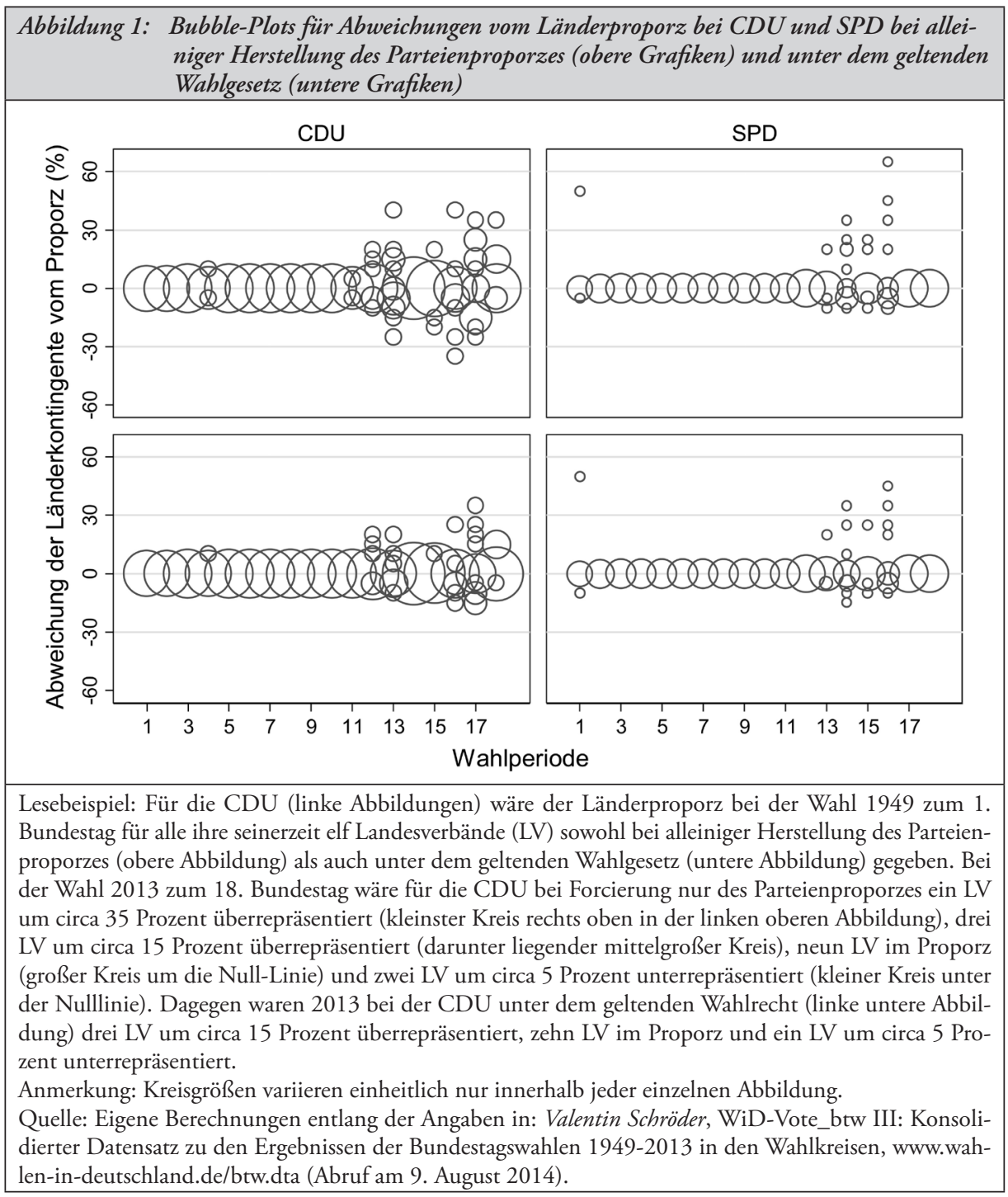

gesetzliche Sitzzahl und für die Direktmandate die tatsächlich erreichten Werte verwendet. Wenn ein Landesverband mit seinem Mandatskontingent genau im Länderproporz lag, wird er in der Abbildung für die jeweilige Wahlperiode als Kreis auf der Nulllinie darge-

der Oberverteilung abgezogen. Für die Wahl 1949 wurde, ebenfalls zur Herstellung von Vergleichbarkeit, außerdem die gesetzliche Sitzzahl auf 484 festgelegt. Seinerzeit lag die Summe der Landeskontingente als gesetzliche Sitzzahl bei 400, wobei 242 Direktmandate und 158 Listenmandate vorgesehen waren. Diese Zahl wurde hier um 84 erhöht, um am Anfang der Berechnung erst einmal von gleich vielen Direkt- und Listenmandaten auszugehen, wie das seit 1953 der Fall ist. 
stellt. Weicht er davon ab, wird er um den entsprechenden Prozentwert (bezogen auf den Anteil überzähliger oder fehlender Mandate an den ihm im Proporz zustehenden Sitzen) nach oben oder unten verschoben dargestellt. ${ }^{28}$ Die Anzahl der Landesverbände mit ähnlich großen Abweichungen wird durch Unterschiede in der Größe der Kreise dargestellt. Je größer ein Kreis in der Abbildung ist, desto mehr Landesverbände sind um den entsprechenden Prozentwert über- oder unterrepräsentiert. So stellen beispielsweise bei der SPD in der 1. Wahlperiode die beiden kleinen Kreise je ein über- und ein unterrepräsentiertes Landeskontingent dar und der große Kreis auf der Nulllinie die übrigen zehn zumindest nahezu im Proporz vertretenen SPD-Landeskontingente. Wenn wiederum alle Landeskontingente nahezu genau dem Proporz entsprechen - bei der CDU zum Beispiel in der 1 . Wahlperiode - gibt es nur einen sehr großen Kreis. Weichen sie voneinander ab, gibt es mehrere kleinere Kreise, die wiederum jeweils umso größer sind, je mehr Landesverbände um den entsprechenden Wert vom Proporz abweichen. Bei allen vier Simulationsserien wird das Bild ab der 13. Wahlperiode (1994) diffuser: Die Kontingente vieler Landesverbände weichen nun oft vom Länderproporz ab. Nur bei der SPD kommt es mangels Überhangmandaten ab 2009 wieder zu dessen durchgehender Einhaltung.

Das Ausmaß der Abweichungen vom Länderproporz ist bei den Simulationen unter dem geltenden Wahlgesetz im Vergleich zu dem nur den Parteienproporz sichernden Regime aber deutlich abgemildert, vor allem bei der zuletzt besonders stark mit Überhangmandaten versehenen CDU. Dort bewegen sie sich auch für die Wahlen von 2005 und 2009, in denen die elektorale Konzentration des deutschen Parteiensystems besonders gering war, in einem Intervall von rund $+/$ - 30 Prozent und streuen innerhalb dieses Intervalls generell weniger als unter dem mandatsminimierenden Regime. Das liegt an der Bundestagsvergrößerung. Sie wirkt der Kompensation doppelt entgegen. Erstens fallen Überhangmandate nun weniger ins Gewicht oder verschwinden parteiintern sogar; und zweitens stehen einfach mehr Mandate für die Annäherung an den Länderproporz zur Verfügung. Dieser Zusammenhang selbst überrascht mit Blick auf die Argumentation oben zwar nicht. Die durchaus nennenswerte Größenordnung der sonst eintretenden Verzerrung wird aber erst jetzt deutlich.

Die Bundestagsvergrößerung durch das neue Wahlrecht erfüllt also zumindest parteiintern durchaus einen Zweck: Sie mildert Disproportionalitäten zwischen den Landesverbänden im Bundestag deutlich ab. In absoluten Mandatszahlen profitieren davon zwar besonders die großen Landesverbände. ${ }^{29}$ Relativ gesehen - und für den Länderproporz entscheidender - erhalten aber gerade kleinere Landesverbände, die außerdem in der Regel kaum Direktmandate erzielten (bei der CDU zum Beispiel Brandenburg, Bremen, Hamburg), eine gewisse Absicherung, überhaupt im Bundestag vertreten zu sein.

Dieser Vorteil des geltenden Wahlgesetzes geht aber mit einem beachtlichen Mandatsaufwuchs einher. Zudem besteht nur ein sehr loser Zusammenhang zwischen seiner Höhe und Verzerrungen des Länderproporzes durch Überhangmandate, wie Abbildung 2 zeigt.

28 Um die Grafik nicht vollkommen unübersichtlich werden zu lassen, wurden Abweichungen in Fünf-Prozent-Schritten zu Kategorien zusammengefasst, wobei die Nulllinie als Ausgangspunkt diente. Abweichungen vom Länderproporz um $+/-2,5$ Prozent fallen dadurch grafisch in die "Null-Abweichungskategorie“.

29 Vgl. auch Joachim Behnke, a.a.O. (Fn. 2). 


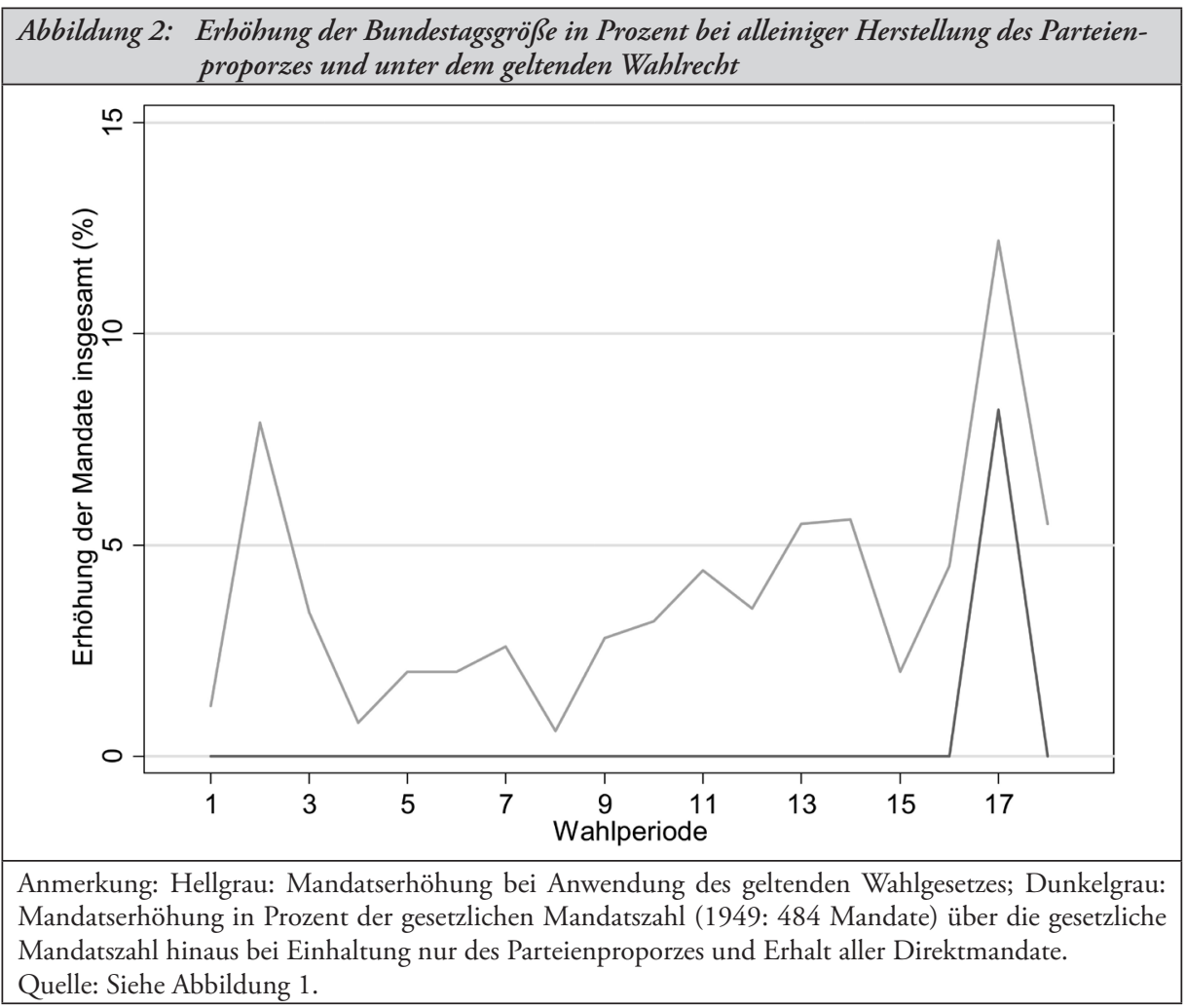

Das ist nicht überraschend, denn es besteht nur dann ein Zusammenhang zwischen beidem, wenn die Bundestagsvergrößerung durch Direktmandate einer bundesweit kandidierenden Partei verursacht wird. So wäre es in den Simulationen aber nur dreimal gekommen, 2002 und 2005 durch die SPD-Mindestsitze und 2009 durch die der CDU. Sonst hätte die Vergrößerung nahezu immer aus den Mindestsitzen der CSU oder der PDS resultiert. ${ }^{30}$ Da bei diesen beiden relativ kleinen Parteien zum Ausgleich ihrer Überrepräsentati-

30 Zur Vergrößerung wäre es durch die Mandatskontingente der CSU (1953, 1961 bis 1990, 2013), der PDS (1994 bis 1998), der FDP (1949) und der DP (1957) gekommen. Die Ursachen für die besonders häufige Überrepräsentation von CSU und PDS wären wiederum besonders niedrige Wahlbeteiligungsquoten gepaart mit vergleichsweise hohen Stimmenanteilen für bei der Sitzzuteilung nicht zu berücksichtigende Parteien und vielen ungültigen Stimmen in den Bundesländern gewesen, in denen diese beiden Parteien die weitaus meisten beziehungsweise alle Stimmen erzielten. In diesen Ländern wären dadurch die Mandatskontingente für den Ersten Schritt der Mandatsverteilung im Vergleich zu groß gewesen. Alle zuteilungsberechtigten Parteien vor Ort hätten in diesen Bundesländern dann erst einmal mehr Sitze erhalten, als ihrem Anteil an den bundesweiten Zweitstimmen entsprochen hätte. Bei der Aufsummierung der Mandate aus den Landeskontingenten für die Parteien wären für die meisten Parteien diese Mehrmandate wiederum durch Mindermandate in den zunächst zu kleinen Landeskontingenten ungefähr ausgeglichen worden. Für CSU und PDS wäre dies aufgrund der Konzentration ihrer Stimmen in den Ländern mit zunächst zu großen Kontingenten aber nicht möglich gewesen, denn dafür fielen bei PDS und CSU andernorts zu wenig (oder eben gar keine) Stimmen an. Seit der Annäherung der 
on beim Parteienproporz besonders viele Mandate an die anderen Parteien verteilt werden müssen, wären auf die CDU- und SPD-Landeslisten zwar besonders viele zusätzliche Sitze entfallen, und dadurch wären direktmandatsbedingte Verzerrungen beim Länderproporz auch besonders effektiv eingedämmt worden, wie ein Vergleich der Abbildungen 1 und 2 für die Wahlen 1990 bis 1998 (12. bis 14. Wahlperiode) ergibt. Aus demselben Grund wäre es aber auch schon in der Zeit davor zur Bundestagsvergrößerung gekommen, also als der Länderproporz schon von vornherein hergestellt worden wäre. ${ }^{31}$ Eine Bundestagsvergrößerung hätte in diesen Fällen also der Einhaltung keines einzigen der drei Repräsentationsprinzipien gedient.

Das neue Wahlgesetz ist folglich als Instrument zur Annäherung an den Länderproporz durch die Generierung von zusätzlichen Sitzen zwar nicht ungeeignet. Aber es ist ineffizient, denn es erzeugt Mehrmandate auch dann, wenn sie dafür nicht notwendig wären. Mit Blick auf den Zielkonflikt von sparsamer Bundestagsgröße und Einhaltung der drei Repräsentationsprinzipien ist diese Ineffizienz also das Kritikwürdige an der Tendenz zur Bundestagsvergrößerung im neuen Wahlgesetz, aber nicht die Tendenz zur Vergrößerung als solche. Sie kann durchaus der Einhaltung eines Repräsentationsprinzips dienen.

\section{Herstellung des Länderproporzes durch direktmandatsorientierte Proporzanpassung und ihre Effizienz}

Da die Verzerrung des Länderproporzes durch Direktmandate im geltenden Wahlrecht nur recht ineffizient eingedämmt wird, geht es nun darum zu untersuchen, wie dies effizienter geschehen kann. Dabei wird unter „effizient“ die geringstmögliche Vergrößerung des Bundestags über die gesetzliche Sitzzahl hinaus bei gleichzeitiger Herstellung eines vollständigen Länderproporzes und unter Einhaltung des Parteienproporzes sowie der Personenwahl in ihrer bestehenden Form (relative Mehrheitswahl in Einpersonen-Wahlkreisen) verstanden. Wenn die Anzahl der verteilten Mandate zumindest nicht allzu weit über der gesetzlichen Sitzzahl von 598 als Zielwert liegen soll, hat das zwei Implikationen: Erstens legt das Ziel der Effizienz die bundesweite Anzahl der Direktmandate einer Partei als Kriterium für die Ermittlung ihrer Mindestsitze nahe und nicht, wie bisher, ihre Zweitstimmen in den einzelnen Bundesländern. Zweitens sollte der - mit Blick auf den Zielkonflikt bei der Umsetzung der drei Repräsentationsprinzipien unvermeidliche - Eingriff in die Mehrheitswahlkomponente möglichst gering gehalten werden. Dies bedeutet wiederum Abstriche bei der Effektivität des Verteilungsverfahrens, also bei dem Grad, zu dem ein perfekter Länderproporz erreicht werden kann.

Mit Blick auf die erste Implikation bietet sich das so genannte Pukelsheim-3-Verfahren an, denn dort sind die Direktmandate der Bezugspunkt für jede Bundestagsvergrößerung. ${ }^{32}$

Stimmenanteile in Ost und West für die PDS/Die Linke ab der Wahl 2005 kann dieses Phänomen besonders noch bei der CSU auftreten, und stellte sich auch 2013 wieder ein. Dies (und nicht die vier CDU-Überhangmandate) war die Ursache für die derzeitige Bundestagsvergrößerung von 598 auf 631.

31 Dies ergeben auch die Simulationen entlang von Wahlumfragen im Zeitraum 2009 bis 2013 bei Philipp Weinmann, a.a.O. (Fn. 3), S. 727 ff.

32 Vgl. Richard Pfeifer / Daniel Lübbert / Kai-Friederike Oelbermann / Friedrich Pukelsheim, a.a.O. (Fn. 9). 
Bei diesem Verfahren wird zuerst eine testweise Oberverteilung vorgenommen. In dieser „Anfangszuteilung“ werden erst einmal so viele Mandate im Parteienproporz vergeben, dass die gesetzliche Mandatszahl voll ausgeschöpft wird. Anschließend werden für jede Partei ihre Direktmandate bundesweit aufsummiert und für jede Partei mit mindestens zwei Landeslisten zudem mit dem Faktor 1,1 multipliziert (die „Zwischenrechnung“). So ergibt sich für jede Partei eine Mindestsitzzahl, die sie auf jeden Fall erhält, einschließlich eines Mandatsaufschlags als Puffer für den Ausgleich von Verzerrungen beim Länderproporz. Falls für eine Partei diese Mindestsitzzahl höher ist als ihre Sitzzahl laut Anfangszuteilung, erhalten die anderen Parteien so viele zusätzliche Mandate, dass der Parteienproporz abermals hergestellt ist („Proporzanpassung“). Die Partei, durch deren Mindestsitzzahl die Proporzanpassung ausgelöst wird, wird hier „Ankerpartei“ genannt. Da der Mandatsaufschlag keiner Partei mit nur einem Landesverband zuteil wird, kommt es zu keiner „unnötigen“ Mandatserhöhung durch eine Ankerpartei, die von Länderproporzverzerrungen nicht betroffen sein kann. Den Karlsruher Kriterien wäre mit dem Pukelsheim-3-Verfahren also Genüge getan, denn mit dieser direktmandatsorientierten Proporzanpassung sind Personenwahl und Parteienproporz generell gewahrt. ${ }^{33}$ Wenn keine Partei mehr Mindestsitze erzielt hat, als ihr laut Anfangszuteilung zustehen - wenn es also keine Ankerpartei gibt - könnte es insoweit außerdem bei 598 Bundestagsmandaten bleiben.

Unter Umständen ist dann aber der Länderproporz dennoch verfehlt. Der zehnprozentige Aufschlag stellt zwar einerseits sicher, dass zu dessen Annäherung Mandatsansprüche bestehen. Andererseits ist aber nicht gewährt, dass dieses Kontingent für die Herstellung des Länderproporzes ausreicht. Folglich ist dieses Verfahren zwar effizienter als das geltende Wahlrecht - es kommt vor allem dann zur Bundestagsvergrößerung, wenn eine Partei mehr Direktmandate erzielt, als sie laut Parteienproporz an der gesetzlichen Sitzzahl beanspruchen kann. Aber ein perfekter Länderproporz ist durch den Zehn-Prozent-Aufschlag ebenfalls nicht garantiert.

Nun könnte der Länderproporz mit dem Pukelsheim-3-Verfahren forciert werden, indem die Höhe des Mandatsaufschlags heraufgesetzt wird. Dadurch würde es jedoch wieder an Effizienz verlieren, denn der Aufschlag wird ja immer fällig, also auch wenn gar keine Überhangmandate entstehen. ${ }^{34}$ Zudem gerät der Zielwert bei den insgesamt zu verteilenden Sitzen in umso weitere Ferne, je akkurater der Länderproporz per Mandatsaufschlag hergestellt wird. So wäre bei der Bundestagswahl 2009 ein Faktor von 1,3 erforderlich gewesen, um 234 Mandate für die CDU zu erzeugen, mit denen alle ihre Landesverbände im Proporz hätten bedient werden können. Dem Bundestag hätten dann jedoch 806 Abgeordnete angehört. ${ }^{35}$

Hier tritt der Zielkonflikt zwischen Umsetzung der personalisiert-territorialen Repräsentation und Einhaltung des Länderproporzes auf der einen Seite und sparsamer Mandatszahl auf der anderen zutage. Ein alternativer Weg zum Länderproporz bestünde entsprechend

33 Damit kann auch kein negatives Stimmengewicht in dem vom Bundesverfassungsgericht monierten Sinne auftreten.

34 Dazu kommt es immer dann, wenn die Ankerpartei mindestens so viele Direktmandate erzielt, dass ihre Summe zuzüglich Direktmandatsaufschlag über ihrer Sitzzahl laut Anfangszuteilung liegt. Erzielt sie folglich zum Beispiel exakt so viele Direktmandate, wie ihr laut Anfangszuteilung zustehen, und entspricht deren Verteilung über die Bundesländer auch dem Länderproporz, vergrößert sich der Bundestag beim Pukelsheim-3-Verfahren um zehn Prozent.

35 Diese Zahlen ergeben sich aus den Simulationen unten. 
darin, von vornherein weniger Direktmandate zu vergeben. Für das Beispiel der Wahl 2009 hätte etwa ihre Kürzung auf rund 70 Prozent des jetzigen Bestands den Länderproporz in der CDU schon bei insgesamt 598 Mandaten gesichert. ${ }^{36}$ Anstelle von 299 würde es dann aber nur noch rund 210 Wahlkreise geben. Der Proporz hätte also zumindest in dem Fall, in dem bislang die deutlichsten parteiinternen Proporzverzerrungen auftraten, nur auf Kosten einer kompletten Neujustierung der Personenwahl, etwa im Zuge einer umfangreichen Wahlkreisreform, hergestellt werden können.

Wie diese Beispiele zeigen, sind die Einhaltung von Personenwahl, Parteienproporz, Länderproporz und gesetzlicher Mandatszahl von 598 unter den derzeit geltenden wahlrechtlichen Parametern ab einem bestimmten Niveau elektoraler Dekonzentration unmöglich. Mindestens eines dieser Ziele wird beim Streben nach den anderen dann immer verfehlt, und zwar deutlich. Die Entwicklung des Bundestagswahlrechts zeigt aber, dass zumindest kleinere Abweichungen von einer perfekten Umsetzung jedes dieser Anliegen die Legitimität des gesamten Wahlsystems nicht beeinträchtigen. Namentlich die Abweichung vom Parteienproporz durch Überhangmandate blieb ja gerade deshalb jahrzehntelang unbeachtet, weil sie diesen Proporz nie in für die Parteien handlungsleitender Weise tangierte. Die oben dargelegte Argumentation der Unionsfraktion bei den Verhandlungen zum geltenden Wahlgesetz zeigt zudem, dass auch der Länderproporz nicht perfekt sein muss.

Dieser Pragmatismus legt es wiederum nahe, Auswirkungen der Änderung einzelner Elemente des Bundestagswahlrechts zunächst empirisch zu untersuchen und zu ermitteln, inwieweit die Umsetzung der drei Prinzipien von solchen Änderungen tatsächlich betroffen wäre. Daher wird nun mit einer weiteren Serie von Simulationen bestimmt, wie stark sich die Verschiebung des Gewichts auf eines der beiden Ziele (Prinzipieneinhaltung vs. Mandatssparsamkeit) jeweils auf die Umsetzung des anderen ausgewirkt hätte. Dafür wird die Mandatsverteilung für die vier Bundestagswahlen 2002 bis 2013 entlang der direktmandatsorientierten Proporzanpassung untersucht. 2002 bestand mit rund 38 Prozent der Zweitstimmen für die SPD und rund 30 Prozent für die CDU nahezu noch die recht hohe elektorale Konzentration im Parteiensystem, bei der das Anfallen von Überhangmandaten bei bundesweiter Verrechnung der Direktmandate unwahrscheinlich ist. ${ }^{37}$ Zudem waren die Direktmandate ausreichend gleichmäßig zwischen den Parteien verteilt, so dass der Anteil pro Partei vergleichsweise niedrig war und Länderproporzverzerrungen durch Überhangmandate damit nur in kleinem Umfang zu erwarten sind. 2005 war dies durch die fortschreitende elektorale Dekonzentration nicht mehr der Fall: Während nach wie vor nahezu alle Direktmandate ungefähr gleichmäßig auf CDU ${ }^{38}$ oder SPD entfielen, sank die Zahl ih-

36 Die CDU erzielte damals 37 Direktmandate in Baden-Württemberg. Um eine Überrepräsentation dieses Landesverbands (und entsprechende Unterrepräsentation anderer Landesverbände) zu vermeiden, hätte er bei einem Anspruch von insgesamt 173 Mandaten höchsten 27 erhalten, also nur knapp 73 Prozent der dort angefallenen Mandate. Rundungserfordernisse bei 15 CDU-Landeskontingenten weisen dann auf die Zahl 70.

37 Vgl. Joachim Behnke, Ein integrales Modell der Ursachen von Überhangmandaten, in: PVS, 44. Jg. (2003), H. 1, S. 41 - 65, S. 50. Die CSU wird hier nicht weiter untersucht, weil für sie keine Verzerrung des Länderproporzes auftreten kann. Entsprechend ist, da die CDU ja nur in 15 Ländern antritt, zu beachten, dass um rund acht Prozent niedrigere Prozentanteile und Mandatszahlen der CDU gegenüber der SPD ungefähr die bundesweite Parität zwischen CDU/CSU und SPD bedeuten.

38 In Bayern die CSU, vgl. Fn. 10. 
rer Listenmandate drastisch. Entsprechend sollte der Länderproporz nun sichtbar unter Druck geraten. 2009 kam es durch den Absturz der SPD auf 23 Prozent dann nicht nur zur bislang höchsten Dekonzentration des Parteiensystems, sondern auch zu einer Ballung der Direktmandate bei der CDU. Für sie müssten sich dadurch bei bundesweiter Verrechnung besonders gravierende Abweichungen vom Länderproporz zeigen, während dies für die SPD unproblematisch sein sollte. 2013 schließlich treffen wir diese Ballung bei der CDU sogar noch deutlicher an, diesmal jedoch bei über 34 Prozent der Zweitstimmen und damit erneut in einem Umfeld, in dem Überhangmandate bei bundesweiter Verrechnung kaum anfallen und der Direktmandatsanteil an allen CDU-Sitzen den Länderproporz daher kaum verzerren sollte. Die vier Wahlen spiegeln deshalb die Konstellationen wider, unter denen Überhangmandate bei bundesweiter Verrechnung gehäuft zustande kommen können (2005, 2009) oder nicht $(2002,2013)$ und sich bei gehäuftem Auftreten der Direktmandatsanteil auf den Länderproporz einer (2009) oder mehrerer (2005) Parteien auswirken kann.

Das BVerfG-Urteil lässt für die Parameter der Simulationen bereits den Überhangmandaten in Form des alten Wahlrechts und damit einer Verzerrung des Parteienproporzes praktisch keinen Raum mehr. Daher wird bei den Simulationen der Parteienproporz immer so fixiert, wie er laut Zweitstimmen-Ergebnis in Mandate umzusetzen ist. Personalisiert-territoriale Repräsentation und Bundestagsgröße werden hingegen als unabhängige Variablen behandelt, deren Ausprägungen schrittweise verändert werden. Der Grad, zu dem der Länderproporz eingehalten wird, dient als abhängige Variable. Da dieser nur bei Parteien verzerrt sein kann, die in nennenswertem Umfang Direktmandate erzielen, werden zudem nur Mandatsveränderungen bei CDU und SPD sowie die Bundestagsgröße insgesamt betrachtet.

Die Umsetzung der Personenwahl wird variiert, indem die Zahl der insgesamt zu vergebenden Direktmandate vom existierenden Stand aus bis auf die Hälfte bei der jeweiligen Wahl abgesenkt wird. Dies wird in elf Zweieinhalb-Prozent-Schritten getan (von 50 Prozent aller Mandate als Ist-Zustand auf 25 Prozent). Dafür wird bei jedem Schritt und für jede Wahl zuerst die Direktmandatszahl insgesamt abgesenkt und dann per Sainte-Laguël Schepers-Divisorverfahren auf die Bundesländer verteilt. Innerhalb der Länder werden diese Mandate dann, ebenfalls per Sainte-Laguë/Schepers-Divisorverfahren, auf die Parteien verteilt, und zwar im Verhältnis der tatsächlich erzielten Direktmandate. ${ }^{39}$

39 Diesem Vorgehen liegt die Annahme zugrunde, dass ein Neuzuschnitt von Wahlkreisen für sich genommen keine so großen Konsequenzen (für das individuelle Wahlverhalten oder durch „gerrymandering") hat, dass dadurch innerhalb eines Bundeslandes die Wahrscheinlichkeiten einzelner Parteien, Direktmandate zu erzielen, systematisch tangiert werden. Unter dieser Prämisse wurden zudem zwei Probleme bewältigt, die sich aus den tatsächlichen Direktmandaten als Ausgangsgrößen ergaben. Erstens versagt das Sainte-Laguë/Schepers-Divisorverfahren als eindeutiger Zuteilungsmechanismus, wenn ein Mandatskontingent auf Parteien mit jeweils gleich vielen Stimmen (hier: gleich vielen Direktmandaten) verteilt werden soll, das sich nicht ganzzahlig in genau so viele gleich große Teile aufteilen lässt, wie es solche Parteien gibt. Für solche „ties“ sieht das deutsche Wahlgesetz deshalb ein Losverfahren vor (\$6 (2), S. 4 BWG). Zweitens besteht dieses Problem auch in Fällen, in denen Parteien zwar unterschiedlich viele Stimmen erzielt haben, in denen aber die Stimmen geteilt durch den Zuteilungsdivisor bei der Anfangszuteilung für zwei oder mehr Parteien den gleichen Quotienten ergeben. Auch dann erhält man bei nicht ganzzahlig in entsprechend große Teile teilbaren Kontingenten keine eindeutige Zuteilung. In den Simulationen wurde für diese Fälle eine Rangfolge der Parteien nach ihren landesweiten Zweitstimmen gebildet. Von jedem Rangplatz wurde anschließend der Wert 1 abgezogen, die Differenz durch 
Die Bundestagsgröße als zweite Variable wird in den Simulationen zum einen direkt verändert, indem das Pukelsheim-3-Verfahren mit Mandatsaufschlägen auf die Direktmandatssummen der beiden Parteien von null bis fünfzig Prozent (das heißt, mit Faktoren von 1 bis 1,5) durchgeführt wird. Dabei wird der Aufschlag ebenfalls in elf Schritten um je fünf Prozent erhöht. Durch die Verminderung des Direktmandatsanteils wird die Bundestagsgröße zum anderen ebenfalls variiert, allerdings nur indirekt. Denn je nachdem, wie viele Direktmandate auf die Parteien entfallen, kann es beim Pukelsheim-3-Verfahren durch die Proporzanpassung nach der Zwischenrechnung ebenfalls zur Bundestagsvergrößerung kommen. Je weniger Direktmandate wiederum zu verteilen sind, desto weniger davon kann eine einzelne Partei erzielen, desto geringer ist ihre Direktmandatssumme, desto geringer ist die Wahrscheinlichkeit, dass diese Summe (zuzüglich Aufschlag) über ihrem Mandatsanspruch laut Anfangszuteilung liegt und desto unwahrscheinlicher ist schließlich eine Bundestagsvergrößerung.

Entlang der schrittweisen Veränderungen von Mandatsaufschlag und Direktmandatsanteil bei fixer gesetzlicher Mandatszahl lässt sich schließlich das Ausmaß der LänderproporzVerzerrung als abhängiger Variable beobachten. Wie schon oben, wird diese Verzerrung auch hier als durchschnittliche Abweichung des Mandatsanteils eines Landesverbands von dem ihm unter perfektem Proporz zustehenden Anteil an allen Sitzen seiner Partei operationalisiert. Für jede Bundestagswahl ergeben sich durch die jeweils elf Schritte bei den Variationen von Mandatsaufschlag und Direktmandatsanteil insgesamt 121 Simulationen. Die resultierenden 484 Simulationsergebnisse für die vier Wahlen 2002, 2005, 2009 und 2013 sind in den Abbildungen 3, 4 und 5 grafisch dargestellt.

Die Veränderung der Bundestagsgröße wird in Abbildung 3 je nach Direktmandatsaufschlag von links nach rechts aufsteigend (0 bis 50 Prozent) und nach Direktmandatsanteil von unten nach oben absteigend (50 bis 25 Prozent) angegeben. Zur Veranschaulichung sind außerdem Kreise eingezeichnet, die umso größer sind, je mehr Mandate über die gesetzliche Mandatszahl (598) hinaus anfallen. Die Zahlen geben die Anzahl der zusätzlichen Mandate an. Fallen solche laut Simulation nicht an, bleibt der entsprechende Eintrag leer. Beispielsweise wären bei der Wahl 2002 beim derzeitigen Direktmandatsanteil (50 Prozent) und einem Aufschlag von 50 Prozent insgesamt 598+24=622 Bundestagssitze entstanden.

Die Simulation für die 18. Wahlperiode (2013) als direkter Vergleich mit dem geltenden Wahlgesetz demonstriert die deutlich effizientere Mandatsallokation des Pukelsheim-3-Verfahrens: Selbst bei dem geltenden Direktmandatsanteil wäre es bei 598 Mandaten geblieben. ${ }^{40}$ In den Simulationen wird für die anderen drei Wahlen zudem deutlich, dass eine Bundestagsvergrößerung insbesondere davon abhängt, wie viele Direktmandate die Ankerpartei im Verhältnis zu den anderen Parteien erzielt. So wäre es 2002 und 2005, als keine Partei ein Direktmandatsübergewicht erlangte, auch bei einem fünfzigprozentigen Aufschlag höchstens zu 24 Mehrmandaten gekommen. Dagegen tritt bei der Simulation für 2009 für den derzeitigen Direktmandatsanteil auch bei einem Aufschlag von Null eine

100.000 geteilt und der resultierende Wert von der Direktmandatszahl abgezogen. Dadurch wurden bei allen betroffenen Parteien außer der zweitstimmenstärksten Partei die ursprünglichen Direktmandatssummen im Verhältnis dieser Rangfolge jeweils minimal reduziert. Entlang dieser Rangfolge konnten alle „ties“ aufgelöst werden. Das betraf im Zeitraum 2002 bis 2013 die jeweils drei Direktmandate für SPD und CDU in Hamburg bei der Bundestagswahl 2009. Gemäß Zweitstimmenrangfolge erhielt die CDU bei dem dortigen „ties“ ein Mandat mehr als die SPD.

40 Vgl. auch Philipp Weinmann, a.a.O. (Fn. 3). 


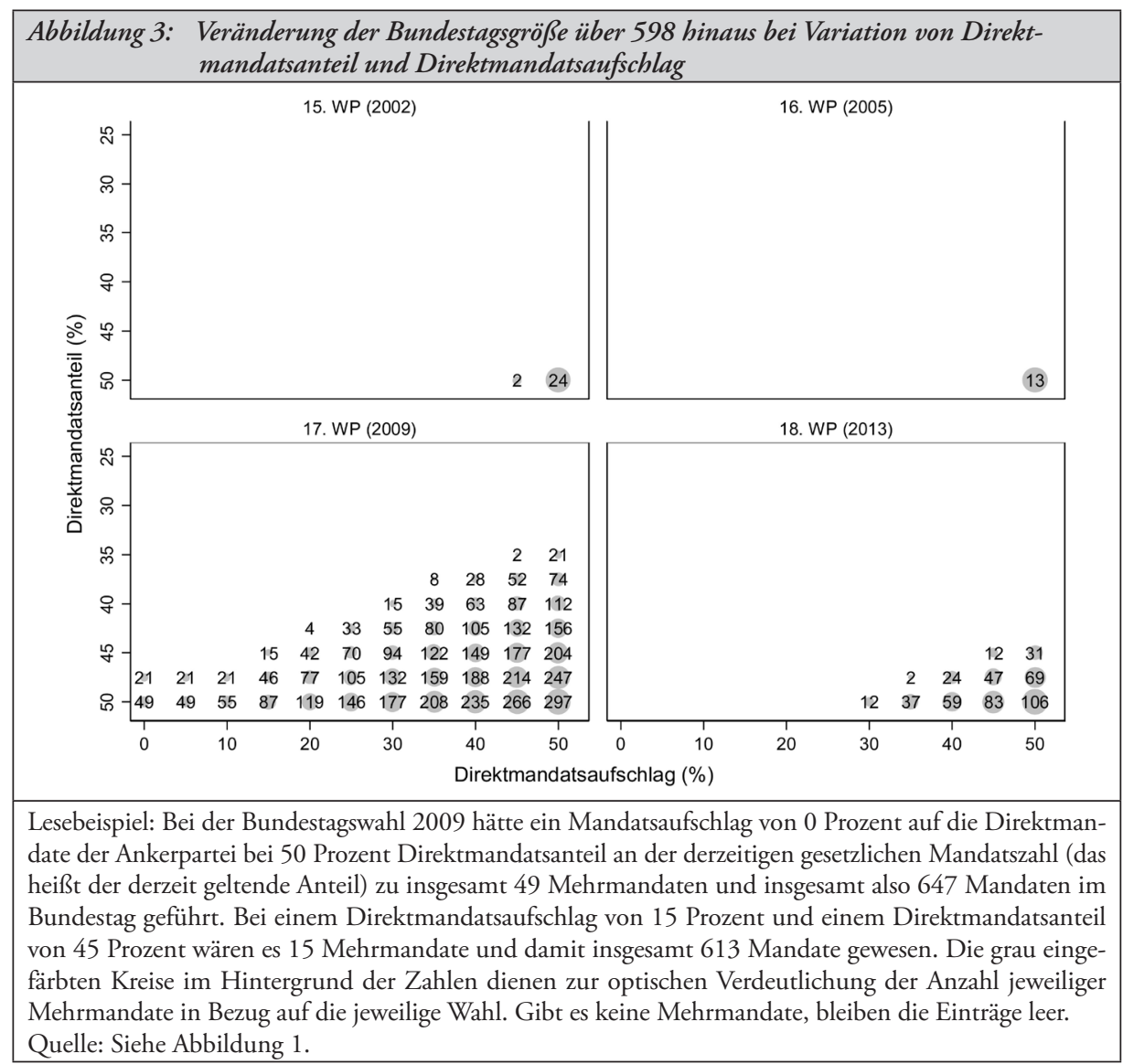

Vergrößerung um 49 Mandate ein. ${ }^{41}$ Auf der anderen Seite mildert schon eine sehr moderate Verringerung des Direktmandatsanteils an der gesetzlichen Sitzzahl die Aufblähungswirkung der Aufschläge deutlich ab. So wäre die Bundestagsgröße bei einem Direktmandatsanteil von 45 statt 50 Prozent selbst 2009 mit 640 Mandaten auch bei einem zwanzigprozentigen Aufschlag noch unter der von Pfeifer u.a. ermittelten Zahl für das originäre Pukelsheim-3-Verfahren geblieben. ${ }^{42}$

Gerade gegenläufig zur Bundestagsgröße entwickelt sich in steigenden Mandatsaufschlägen die Annäherung an den Länderproporz (Abbildungen 4 und 5). Je höher die Aufschläge sind, desto eher wird er erreicht. Allerdings hängt dies davon ab, ob durch den Mandatsaufschlag eben auch eine Bundestagsvergrößerung ausgelöst wird, denn wenn es zu keiner Mandatserhöhung über 598 hinaus kommt, kommt es ja auch zu keinen zusätzlichen Listenmandaten. So wäre es beispielsweise in der 16. Wahlperiode (2005) gewesen. Bei 145 SPD- und 106 CDU-Direktmandaten hätte damals erst ein fünfzigprozentiger Direktman-

41 Die Ursache dafür ist die Mindestsitzzahl der CSU, die 2009 drei Überhangmandate erzielte.

42 Vgl. Richard Pfeifer / Daniel Lübbert / Kai-Friederike Oelbermann / Friedrich Pukelsheim, a.a.O. (Fn. 9), S. 730. 
datsaufschlag die SPD zur Ankerpartei gemacht und im Zuge der Proporzanpassung trotzdem nur eine Erhöhung um 13 Mandate verursacht (siehe Abbildung 3). Gleichzeitig hatten CDU und SPD gemessen an ihren Zweitstimmenanteilen jeweils so viele Direktmandate erzielt, dass sie mit je rund sechs zusätzlichen Listenmandaten auch dann noch den Länderproporz im Mittel um sechs beziehungsweise zwölf Prozent verfehlt hätten. Für eine Annäherung an den Länderproporz hätte es entsprechend höherer Aufschläge und damit wiederum einer massiven Bundestagsvergrößerung bedurft. Mandatsaufschläge allein erweisen sich in den Simulationen also vor allem dann als ein eher plumpes Instrument zur Annäherung an den Länderproporz, wenn die Direktmandate zwischen CDU und SPD in ihrem Größenverhältnis aufgeteilt werden.

Umso bemerkenswerter ist deshalb die zügige Annäherung an den Länderproporz entlang einer Verringerung des Direktmandatsanteils. Schon bei einer Senkung von 50 auf 45 Prozent wird er oft perfekt eingehalten, ohne dass dafür überhaupt Mehrmandate erforderlich sind. Mit Blick auf eine Erhöhung der Effizienz des Verteilungsmechanismus ergibt sich des-

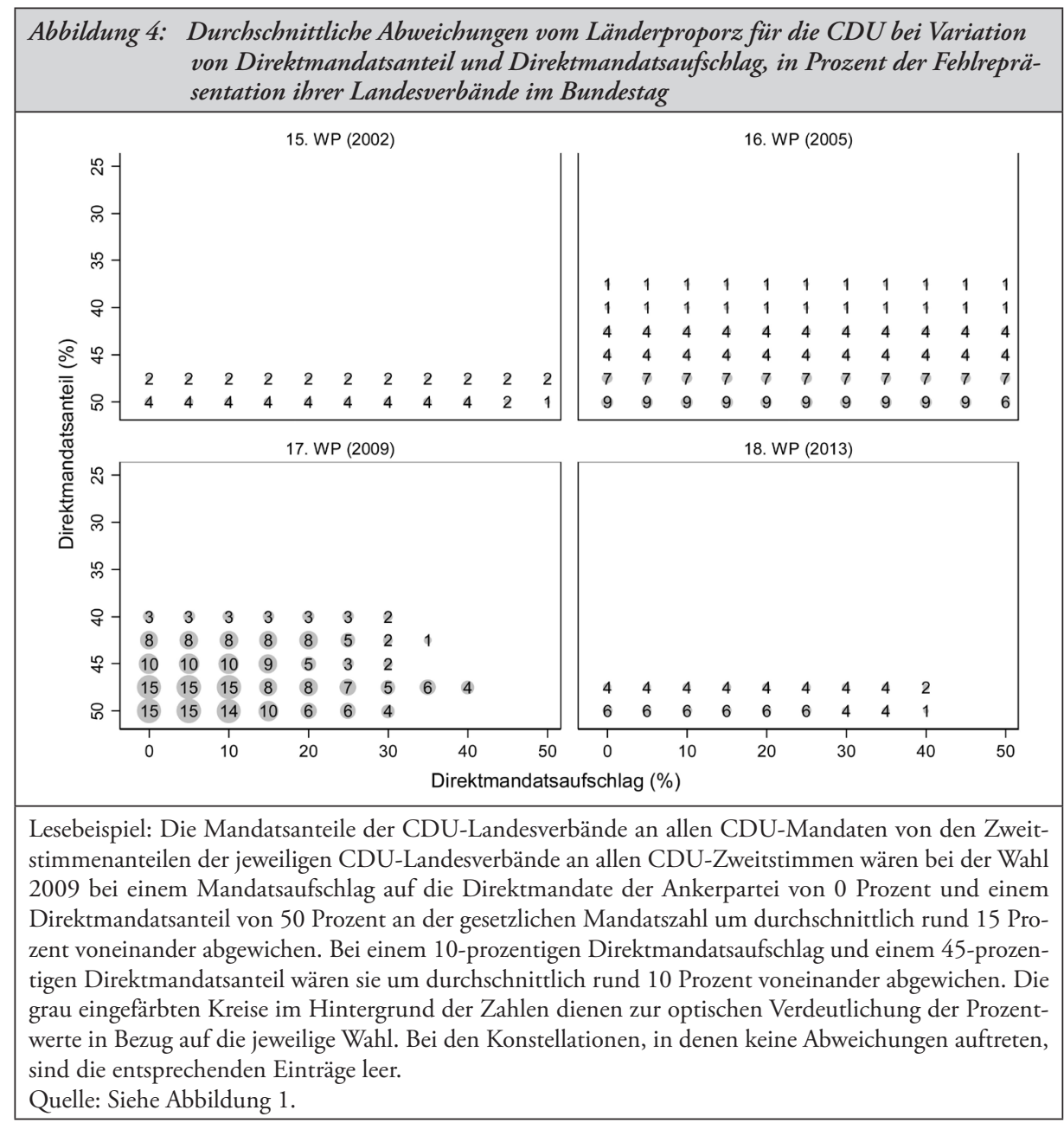




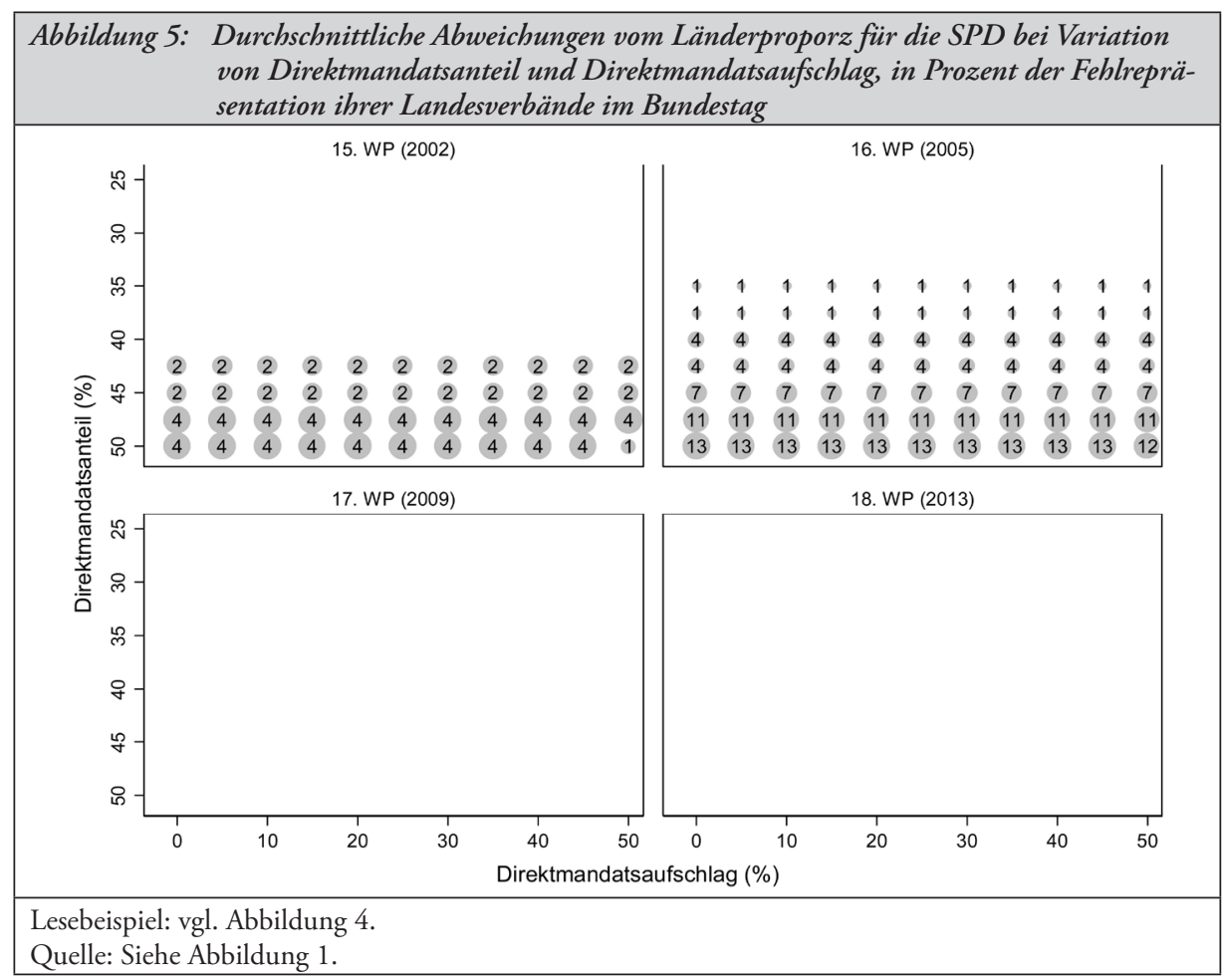

halb in erster Linie eine moderate Absenkung des Direktmandatsanteils. Wenn zudem die Wahl 2009 mit ihrem bislang massivsten Auftreten von Überhangmandaten und Konzentration der Direktmandate bei nur einer Partei als besonders große Herausforderung für die Einhaltung der drei Prinzipien bei möglichst sparsamer Bundestagsgröße verstanden wird, legen die Befunde zudem die Kombination einer Verringerung des Direktmandatsanteils auf 45 statt bislang 50 Prozent und einen Direktmandatsaufschlag von zwanzig Prozent nahe. Dann kommt es bei den Simulationen nie zu Länderproporzverzerrungen über durchschnittlich zehn Prozent. Mit anderen Worten: das geringfügig angepasste Pukelsheim-3-Verfahren verbunden mit einer Wahlkreisreform ungefähr im Rahmen der Reform von 2001 würde genügen, um den Zielkonflikt aus Prinzipieneinhaltung und Mandatsbegrenzung weitgehend oder sogar vollständig zu bewältigen. Damit bleibt der unitarische Charakter des Bundestags bei seiner Zusammensetzung nach Parteistärken im gesamten Bundesvolk (und nicht jeweils in 16 „Landesvölkern“) gewahrt. Gleichzeitig wird das Prinzip des innerparteilich-föderalen Proporzes nahezu perfekt umgesetzt (selbst bei der Wahl 2009, wo dies bei dem ursprünglichen Pukelsheim-3-Modell spürbar verfehlt wird). Zudem werden „übergroße“ Bundestage vermieden. Und schließlich ist dafür nur eine maßvolle Verringerung der Wahlkreisanzahl nötig. Diese müsste keineswegs um 60 oder noch mehr reduziert werden, ${ }^{43}$ und

43 Dies wäre die Konsequenz der Vorschläge von zum Beispiel Behnke und Meyer, vgl. Joachim Behnke, Überhangmandate bei der Bundestagswahl 2009 - das ewige Menetekel, in: PVS, 51. Jg. (2010), H. 3, S. 531 - 552, S. 256; Joachim Behnke, a.a.O. (Fn. 5), S. 686; Hans Meyer, a.a.O. (Fn. 3), S. 211. 
es würde bei weitem zu keinen „Riesenwahlkreisen“ kommen. Entsprechend wird die personalisiert-territoriale Repräsentation nur geringfügig berührt, während der Länderproporz deutlich angenähert werden kann. ${ }^{44}$

Aus den Abbildungen 3 und 4 geht aber auch hervor, dass eine zu geringe Reduzierung des Direktmandatsanteils gerade länderproporzverzerrend wirkt. In der Wahl 2009 hätte beispielsweise die Absenkung auf 47,5 Prozent bei der CDU den Länderproporz stärker verzerrt als der gegenwärtige Direktmandatsanteil. Der Grund dafür ist die unterschiedliche Größe der Bundesländer. Bei den sechs Ländern mit derzeit weniger als zehn Direktmandaten hat eine so geringe Absenkung rundungsbedingt in der Regel keinen Effekt. Gerade dort fallen aber oft Überhangmandate an (zum Beispiel im Saarland und den neuen Bundesländern) und die zugrunde liegenden Direktmandate schlagen in der Summe umso stärker auf den Proporz durch. Eine Wahlkreisreform sollte daher auch nicht zu zaghaft sein.

\section{Konklusion: Plädoyer für eine moderate Senkung der Direktmandate}

Die hier vorgenommenen Simulationen haben demonstriert, dass die geringe Effizienz des derzeitigen Mandatszuteilungsmechanismus durch die direktmandatsorientiere Proporzanpassung entlang des Pukelsheim-3-Modells verbunden mit einer moderaten Absenkung des Direktmandatsanteils entscheidend verbessert werden kann, ohne dass ein massiver Eingriff in die Umsetzung der drei Repräsentationsprinzipien (Parteienproporz, Länderproporz, Personenwahl) vorgenommen werden müsste. Diese Änderungen würden nicht immer zur vollständigen Einhaltung des Länderproporzes führen. Nach wie vor wäre die perfekte Umsetzung aller drei Prinzipien also nicht garantiert. Eine solche „eierlegende Wollmilchsau“ 45 wäre jedoch im Rahmen der Beibehaltung des bisherigen Mischwahlsystems nur zum Preis einer Bundestagsvergrößerung zu haben, die umso drastischer sein müsste, je höher die elektorale Dekonzentration des Parteiensystems und die Ballung der Direktmandate bei nur einer Partei sind.

Eine moderate Senkung des Direktmandatsanteils - die Simulationen legen die Senkung von 299 auf rund 270 Mandate nahe - würde die Umsetzung des Prinzips der personalisiert-territorialen Repräsentation als Teil der mit der Personenwahl verbundenen Verhältniswahl nicht ernsthafter beeinträchtigen, als dies bereits mit der Wahlkreisreform von 2001 geschah. Höchstwahrscheinlich wäre sie für CDU, CSU und SPD mit dem Verzicht auf den einen oder anderen „Erbhof“ verbunden. Dies wäre aber gerade ein sichtbarer Beleg für die Bereitschaft der Parteien, die im Vergleich mit anderen Staaten außerordentlich gut verankerten Repräsentationsprinzipien des deutschen Wahlsystems ${ }^{46}$ auch dann ernst zu nehmen, wenn ihre Einhaltung mit dem Verzicht auf einzelne vermeintlich sichere Wahlkreise einhergeht. Gleichzeitig wäre eine - ebenso moderate - Bundestagsvergrößerung

44 Selbst in Nordrhein-Westfalen als bevölkerungsreichstem Land würde die Direktmandatszahl nur von 64 auf 58 sinken. Durch den weitgehenden Wegfall der Proporzverzerrungen (von denen CDU und SPD dort sonst besonders betroffen wären) ergäbe sich dort zudem ceteris paribus eine Vergrößerung der Landeskontingente dieser Parteien.

45 Suzanne S. Schüttemeyer, Editorial, in: ZParl, 42. Jg. (2012), H. 3, S. 505 - 506, S. 505.

46 Vgl. Eckhard Jesse, Wahlsysteme und Wahlrecht, in: Oscar W. Gabriel / Sabine Kropp (Hrsg.), Die EU-Staaten im Vergleich, Baden-Baden 2008, S. 297 - 322, S. 315. 
über die seinerzeit mit dem Argument der Arbeitsfähigkeit vereinbarte Größe von $600^{47}$ hinaus dann gut begründbar, wenn sie diesen Prinzipien dient.

Nun ist es um das Wahlrecht im Bundestag erst einmal ruhig geworden. Aber, in den Worten von Bundestagspräsident Norbert Lammert in seiner jüngsten Antrittsrede: „[D]a es immer besser ist, sich mit solchen Entwicklungen dann auseinanderzusetzen, wenn die Probleme noch nicht eingetreten sind, spricht manches dafür, dass wir nicht erst nach der nächsten Wahl, sondern rechtzeitig vor der nächsten Wahl noch einmal einen gemeinsamen sorgfältigen Blick auf diese Regelungen werfen. “48

47 Vgl. Deutscher Bundestag, Stenografischer Bericht, 129. Sitzung, Plenarprotokoll 13/129 vom 11. Oktober 1996; Deutscher Bundestag, Zwischenbericht der Reformkommission zur Größe des Deutschen Bundestages, Drs. 13/4560 vom 8. Mai 1996; Deutscher Bundestag, Schlussbericht der Reformkommission zur Größe des Deutschen Bundestages, Drs. 13/7950 vom 17. Juni 1997.

48 Deutscher Bundestag, Stenografischer Bericht, Plenarprotokoll 18/1 vom 22. Oktober 2013, S. 8.

\section{Erfolgswertgleichheit als Maßstab für die Sitzverteilung in Parlamenten}

Fred Hermsdorf

In Wahlgesetzen wird unter anderem geregelt, in welcher Form die abgegebenen Stimmen zur Ermittlung der Sitzverteilung im Parlament herangezogen werden und nach welchen Verfahren bei Verhältniswahlen die entsprechenden Stimmen auf die Parteien verteilt werden. Zur Berechnung dieser Sitzverteilung existieren mehrere verschiedene Methoden (Hare/Niemeyer, St.Laguë/Schepers usw.). Dieser Beitrag bezieht sich allein auf diese Berechnungsverfahren. Der weitere Einfluss der Wahlgesetze auf die zu verteilenden Parlamentssitze wird außer Acht gelassen.

Es ist im Allgemeinen nicht eindeutig möglich, die (große) Anzahl der Wählerstimmen auf die (kleine) Anzahl der Sitze im Parlament proportional abzubilden. Da es keine Teile von Abgeordneten gibt, entsteht ein Spannungsfeld zwischen exakter Proportionalität und Ganzzahligkeit. Es sind politische Entscheidungen zu treffen, wie mit den (zwangsläufig) überraschenden Effekten der berechneten Sitzverteilungen umzugehen ist. Natürlich sind bei allen diesen Entscheidungen die Vorgaben der Verfassung zu beachten.

Die Auswahl des geeigneten Verfahrens hängt somit von den besonderen Anforderungen $\mathrm{ab}$, die in dem betreffenden Wahlgebiet gelten. Liest man etwa in dem bekannten Werk von Balinski und Young ${ }^{1}$, so wird deutlich, dass dort die Überlegungen zur Überwindung dieser Effekte sehr stark geprägt sind von den Bedingungen der Vereinigten Staaten von Amerika im 19. Jahrhundert, vor allem von Bevölkerungswachstum und dem Entstehen neuer Bundesstaaten.

1 Vgl. Michael L. Balinski / H. Peyton Young, Fair Representation - Meeting the Ideal of One Man, One Vote, New Haven / London 1982. 